\title{
EST'UDIO CITOGENÉTICO EN HÍBRIDOS ENTRE UNA ESPECIE OCTOPLOIDE, TURNERA AURELII Y DOS DIPLOIDES, T. CAERULEA Y T. JOELII
}

\author{
por AVELIANO FERNÁNDEZ1
}

\section{Summary}

\begin{abstract}
Hybridizations were made between $T$. aurelii, $2 n=8 x=40$, and two diploid species $(2 n=2 x=10)$, $T$. caerulea and $T$. joelii. The hybrids were studied cytologically to determine their genomic relationship. Two pentaploid hybrids were obtained with $2 n=5 x=25$. Meiosis in the T. aurelii $x T$. caerulea hybrid was irregular with numerous laggards and some bridges; mean pairing relationships were $16.37 \mathrm{I}, 4.01 \mathrm{II}$ and $0.19 \mathrm{III}$. T. aurelii $\times$ T. joelii hybrid showed very irregular cells with numerous laggards and bridges as well as unreduced gametes. Mean pairing relationship were $17.49 \mathrm{I}, 3.32 \mathrm{II}, 0.26 \mathrm{II}$ and $0.01 \mathrm{IV}$. In previous papers the genomic formulas $\mathrm{A}^{\mathrm{a}} \mathrm{A}^{\mathrm{a}} \mathrm{A}^{\circ} \mathrm{A}^{\circ} \mathrm{BBB} \mathrm{B}^{\circ} \mathrm{B}^{\circ}$ and $\mathrm{CC}$ were proposed for $T$. aurelii and $T$. caerulea respectively. Based on the chromosomes associations found in both hybrids, DD is proposed for T. joelii because it has a different genome. Probably, the presence of bivalents in both hybrids may be due to the pairing among homoeologous chromosomes of the mother plant $T$. aurelii, which is a segmental allooctoploid.
\end{abstract}

\section{Introducción}

El género Turnera que cuenta con nueve series (Urban, 1883), presenta tres números básicos $x=5, x=7$ y $x=13$. La serie Turnera (=Canaligerae) es la única con $\mathrm{x}=5 \mathrm{y}$ con distintos niveles de ploidía, desde el diploide hasta el octoploide (Fernández, 1987). Desde 1982 se está llevando a cabo un programa de cruzamientos controlados con el que se obtuvieron varios híbridos interespecíficos (Arbo y Fernández, 1987). Como resultado de los estudios citogenéticos de los mismos, se analizaron las relaciones genómicas entre las especies diploides de flores amarillas (Fernández y Arbo, 1989), encontrándose que estas especies poseen genomas homeólogos, pero diferentes de los de las otras especies diploides de flores blanco-azuladas (Fernández y Arbo, 1996). También se estudiaron híbridos entre especies

1 Facultad de Ciencias Exactas y Naturales y Agrimensura (UNNE). Miembro de la Carrera del Investigador Científico (CONICET). Instituto de Botánica del Nordeste, C.C. 209,3400 Corrientes. poliploides y diploides, determinando que las especies poliploides son alopoliploides segmentarios (Fernández y Arbo, 1990, 1993a, 1993b).

El objetivo del presente trabajo es el análisis citogenético de los híbridos interespecíficos de T. aurelii $(2 \mathrm{n}=8 \mathrm{x}=40)$ con $T$. caerulea y $T$. joelii $(2 n=2 x=10)$.

\section{Material y métodos}

Los progenitores se indican con la sigla utilizada en el invernáculo. Los ejemplares testigo se conservan en el herbario del Instituto de Botánica del Nordeste (CTES).

T. aurelii. (Gr8) Paraguay, Cordillera, río Salado, Schinini 22603.

T. caerulea. (C2) Brasil, Piauí, Bom Jesus, Krapovickas 38740.

T. joelii. (Co) Brasil, Bahia, $29 \mathrm{~km} \mathrm{~S}$ de Juazeiro, Krapovickas 38793.

T. aurelii $\times$ T. caerulea. Argentina, Corrientes, Arbo 2704.

T. aurelii $\times$ T. joelii. Argentina, Corrientes, Arbo 2597. 
El procedimiento usado para los cruzamientos está descripto en Arbo y Fernández (1987) e incluye los siguientes pasos: castración de las flores de las plantas madres, polinización con anteras de la planta seleccionada como padre, marcación de la flor madre indicando en el rótulo el progenitor masculino. Las semillas obtenidas se sembraron en macetas individuales y los híbridos se trasplantaron después de desarrollarse el primer par de hojas.

Las preparaciones para estudio de meiosis se hicieron fijando los botones florales con cinco partes de etanol absoluto y una parte de ácido láctico (Fernández, 1973) y coloreando con la técnica de Feulgen. Para mitosis se siguió el mismo procedimiento, previo pretratamiento de las raíces con 8-hidroxiquinoleína $0,002 \mathrm{M}$ durante 3 horas a temperatura de laboratorio, entre 18 a $26^{\circ} \mathrm{C}$. Para estimar el porcentaje de fertilidad de polen se usó la técnica de coloración con carmín-glicerina, contando no menos de 300 granos por flor.

\section{Resultados}

De los cruzamientos realizados entre las tres especies se obtuvieron híbridos con $2 n=5 x=25$ entre $T$. aurelii como progenitor fe- menino y las dos especies diploides como progenitores masculinos, pero los cruzamientos efectuados entre estas últimas dieron resultados negativos.

Los progenitores presentaron meiosis regular, formando siempre bivalentes, y la fertilidad de polen fue cercana al 100 \% (Fernández, 1987).

En el híbrido T. aurelii $\times$ T. caerulea se analizaron $66 \mathrm{CMP}$ en metafase I (Tabla 1), y se encontraron 14 configuraciones diferentes, siendo la más frecuente (25\%) 17 I + 4 II y 15 I +5 II (Fig. 1A). Se observó un trivalente (Fig. 1B) en 19,69\% de las CMP. El promedio de univalentes fue de 16,37 , con un rango de variación de 8 a 25; el de bivalentes fue de 4,01, con un rango de variación de 0 a 7 , y el de trivalentes de 0,19 , con un rango de variación de 0 a 1.

En el híbrido T. aurelii $\times T$. joelii se analizaron 53 CMP (Tabla 1), encontrándose 15 configuraciones diferentes, siendo la más frecuente (30 \%) 17 I + 4 II (Fig. 2A). Se observó un trivalente (Fig. 2B) en $26,41 \%$ de las CMP. El promedio de univalentes fue de 17,49 , con un rango de variación de 8 a 25; el de bivalentes fue de 3,32, con un rango de variación de 0 a 7; el de trivalentes de 0,26, con un rango de

Tabla 1. Configuraciones halladas en los híbridos

\begin{tabular}{|c|c|c|c|c|}
\hline Configuración & $\begin{array}{l}\text { T. aurelii } \\
\text { No. CMP }\end{array}$ & $\begin{array}{l}\text { T. caerulea } \\
\text { porcentaje }\end{array}$ & $\begin{array}{l}\text { T. aurel } \\
\text { No. CMP }\end{array}$ & $\begin{array}{l}\text { T. joelii } \\
\text { porcentaje }\end{array}$ \\
\hline $25 \mathrm{I}$ & 1 & 1,50 & 5 & 9,50 \\
\hline $23 I+1 I I$ & 3 & 4,60 & 1 & 1,90 \\
\hline $21 I+2 I I$ & 3 & 4,60 & 3 & 5,70 \\
\hline $19 I+3 I I$ & 6 & 9,10 & 6 & 11,30 \\
\hline $17 \mathrm{I}+4 \mathrm{II}$ & 17 & 25,70 & 16 & 30,20 \\
\hline $15 \mathrm{I}+5 \mathrm{II}$ & 17 & 25,70 & 5 & 9,50 \\
\hline $13 I+6 I I$ & 3 & 4,60 & 2 & 3,60 \\
\hline $11 \mathrm{I}+7 \mathrm{II}$ & 3 & 4,60 & 1 & 1,90 \\
\hline $20 I+1 I I+1 I I I$ & 1 & 1,50 & 3 & 5,70 \\
\hline $18 \mathrm{I}+2 \mathrm{II}+1 \mathrm{III}$ & 3 & 4,60 & 2 & 3,60 \\
\hline 16 I + 3 II +1 III & 4 & 6,00 & 3 & 5,70 \\
\hline $14 \mathrm{I}+4 \mathrm{II}+1 \mathrm{III}$ & 2 & 3,00 & 3 & 5,70 \\
\hline $12 \mathrm{I}+5 \mathrm{II}+1 \mathrm{III}$ & - & - & 1 & 1,90 \\
\hline $10 I+6 I I+1 I I I$ & 2 & 3,00 & - & - \\
\hline $8 \mathrm{I}+7 \mathrm{II}+1 \mathrm{III}$ & 1 & 1,50 & 1 & 1,90 \\
\hline $12 \mathrm{I}+3 \mathrm{II}+1 \mathrm{III}+1 \mathrm{IV}$ & - & - & 1 & 1,90 \\
\hline Total & 66 & 100 & 53 & 100 \\
\hline
\end{tabular}


Tabla 2. Promedio y variación de las asociaciones cromosómicas en metafase I y fertilidad de polen en híbridos pentaploides.

\begin{tabular}{|c|c|c|c|c|c|c|c|}
\hline Híbrido & $2 n$ & I & II & III & IV & CMP & Fert. \\
\hline T. aurelii $\times$ T, caerulea & $2 x=25$ & $\begin{array}{c}16,37 \pm 0,37 \\
8-25\end{array}$ & $\begin{array}{c}4,01 \pm 0,19 \\
0-7\end{array}$ & $\begin{array}{c}0,19 \pm 0,04 \\
0-1\end{array}$ & & 66 & 7,66 \\
\hline T. aurelii $\times$ T. joilii & $2 x=25$ & $\begin{array}{c}17,49 \pm 0,50 \\
8-25\end{array}$ & $\begin{array}{c}3,32 \pm 0,23 \\
0-7\end{array}$ & $\begin{array}{c}0,26 \pm 0,06 \\
0-1\end{array}$ & $\begin{array}{c}0,01 \pm 0,01 \\
0-1\end{array}$ & 53 & 6,43 \\
\hline
\end{tabular}

variación de 0 a 1, y el de cuadrivalentes de 0,001 , con un rango de variación de 0 a 1 (Tabla 2).

En los dos híbridos se observó el mismo rango de variación para los univalentes, entre 8 y 25. El máximo de bivalentes encontrado en T. aurelii $x$ T. caerulea fue de 8 (Fig. 1C).
En los dos híbridos siempre se observaron cromosomas rezagados (Fig. 1D) en anafase/ telofase I acompañados o no por puentes. En T. aurelii $\times T$. joelii a veces algunas células presentan puentes (Fig. 2C) que persisten hasta la profase II, quedando la célula con los dos núcleos unidos (Fig. 2D).
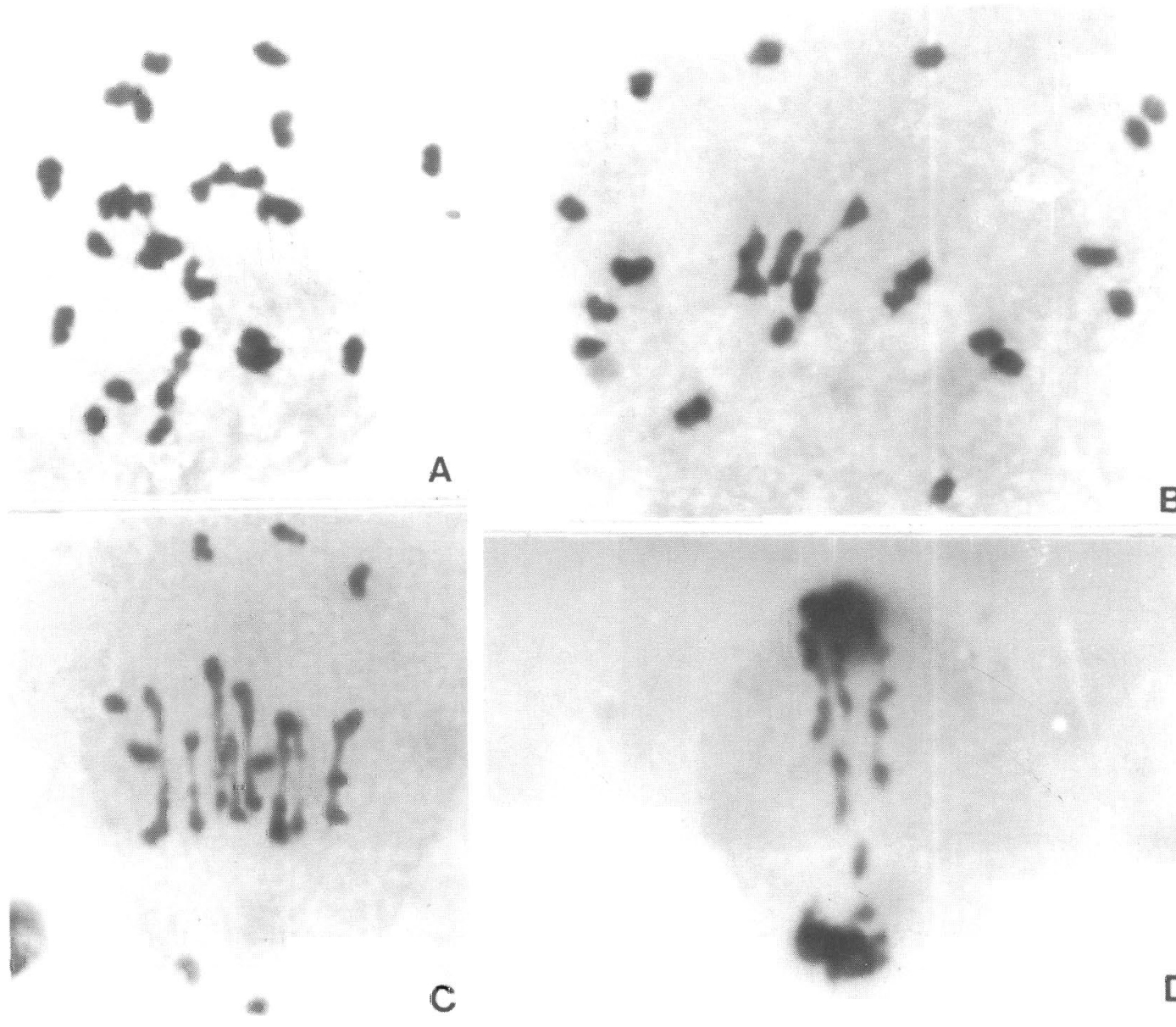

B

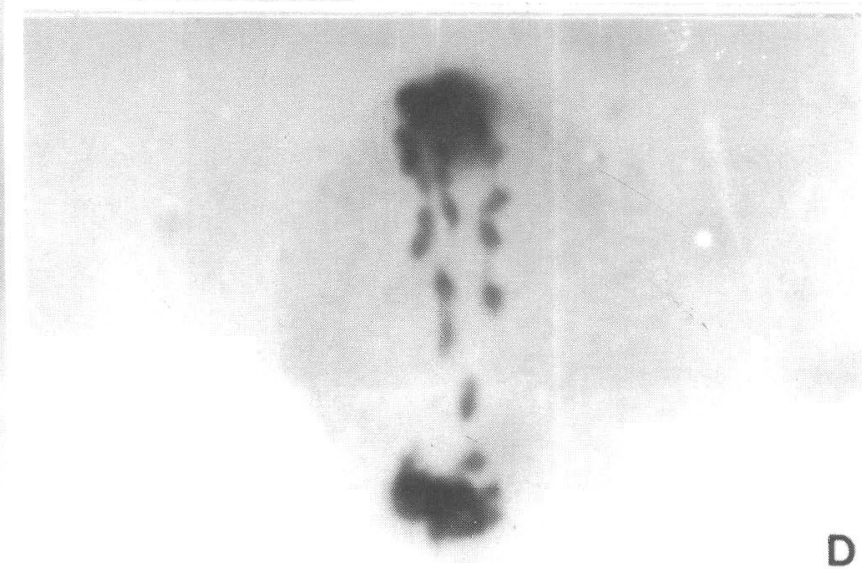

Fig. 1. Meiosis en T. aurelii $x$ T. caerulea, $2 \mathrm{n}=25$. A, metafase I con $15 \mathrm{I}+5 \mathrm{II}$. B, metafase I con $16 \mathrm{I}+3 \mathrm{II}+1 \mathrm{III}$. C, metafase I con $8 \mathrm{I}+8 \mathrm{II}$; en esta célula falta un cromosoma. D, anafase/telofase I con cromosomas rezagados. Escala $=5 \mu \mathrm{m}$. 

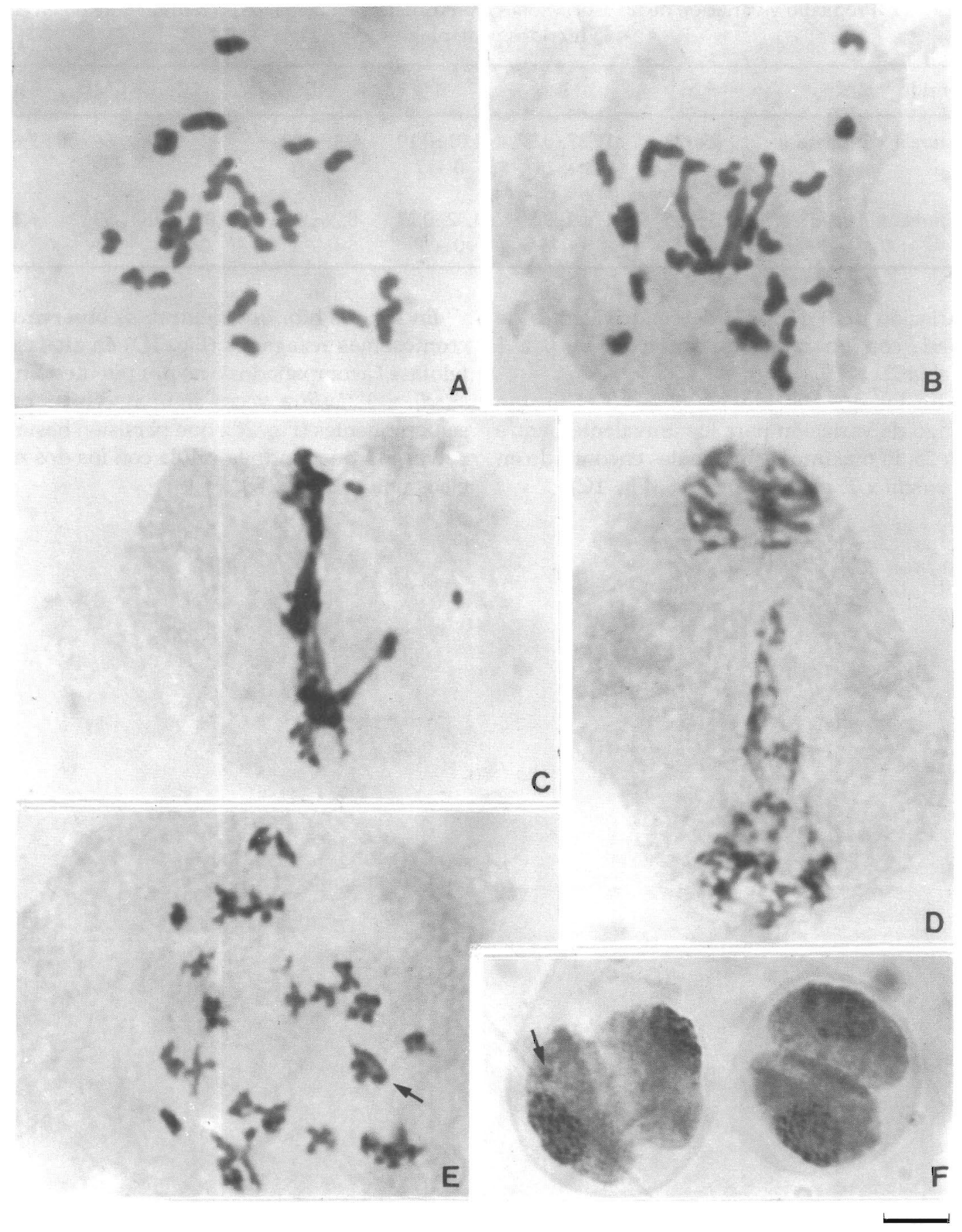

Fig. 2. Meiosis en T. aurelii $x$ T. joelii, $2 \mathrm{n}=25$. A, metafase I con $17 \mathrm{I}+4 \mathrm{II}$. B, metafase I con $18 \mathrm{I}+2 \mathrm{II}+1 \mathrm{III}$. C, anafase I con puentes y fragmentos. D, profase II temprana, ĺos dos núcleos están unidos por puentes remanentes. E, profase II con un solo núcleo con 25 cromosomas, la flecha inđica un cromosoma con las cromátidas aparentemente unidas en los telómeros. F, díadas, en una de ellas se observa un micronúcleo (flecha). Escala $=5 \mu \mathrm{n}$.. 
En algunas células, en là profase II del híbrido $T$. aurelii $\times T$. joelii se observó un solo núcleo con 25 cromosomas (Fig. 2E); aparentemente, estas células no pasaron por la primera división meiótica y como resultado final produjeron díadas (27\%) con 25 cromosomas (Fig. 2F). El 72\% de estas díadas presentaban micronúcleos, mientras que había micronúcleos en $60,79 \%$ de las tétradas. En anafase I y II se observaron cromosomas rezagados y algunos puentes. La fertilidad del polen en los híbridos $T$. aurelii $x T$. caerulea y $T$. aurelii $x T$. joelii fue de $7,66 \%$ y $6,43 \%$ respectivamente.

\section{Discusión}

El cariotipo de las dos especies diploides está formado por 8 cromosomas metacéntricos y 2 submetacéntricos, como en las otras 8 especies diploides analizadas de la serie Turnera (=Canaligerae), fórmula considerada como cariotipo fundamental (Solís Neffa y Fernández, 1993). Las especies se diferencian entre sí por el tamaño y posición del satélite. En T. joelii, el satélite es más grande que en T. caerulea; en ambas especies el par de cromosomas con satélite es el 1 (Solís Neffa, 1996; Solís Neffa y Fernández, 1993). A pesar de tener la misma fórmula cariotípica, hasta el presente fue imposible obtener híbridos entre ellas, lo que indica que están aisladas reproductivamente.

Es importante considerar el comportamiento de la meiosis en los híbridos de $T$. aurelii con las especies diploides. En T. aurelii x T. grandiflora la media de univalentes fue la más elevada (22,58), mientras que en $T$. aurelii $\times T$. krapovickasii y $x T$. concinna la media de univalentes fue alrededor de 8 (Fernández y Arbo, 1993a). En el presente trabajo, la media de univalentes es alrededor de 17 en los dos híbridos. T. caerulea y T. grandiflora pertenecen al grupo de especies diploides con flores blanco-azuladas (Fernández y Arbo, 1996), mientras que $T$. krapovickasii y T. concinna pertenecen al grupo de especies diploides con flores amarillas (Fernández y Arbo, 1989), y ambos grupos pertenecen al complejo T. ulmifolia. $T$. joelii, a pesar de no pertenecer al complejo $T$. ulmifotia, se cruza con las especies poliploides de este complejo, como T. orientalis y T. aurelii (Arbo y Fernández, 1987).

La morfología de los 25 cromosomas observados en profase II (Fig. 2E) en T. aurelii $x T$. joelii, sugiere que en estas células quedó suprimida la meiosis I, y el resultado fue la formación de díadas y de gametos no reducidos, con 25 cromosomas. Probablemente, los pocos granos de polen viables sean los no reducidos. Harlan y de Wet (1975) consideran que este tipo de gametos tiene un papel fundamental en la poliploidización y Asker (1980) opina que la función de los gametos no reducidos, es la de proveer el principal mecanismo para la formación de series poliploides. En Turnera ya se han obtenido híbridos interespecíficos por gametos no reducidos (Fernández y Arbo, 1990).

En trabajos previos se determinaron las fórmulas genómicas de $T$. aurelii $\left(\mathrm{A}^{\mathrm{a}} \mathrm{A}^{\mathrm{a}} \mathrm{A}^{\circ} \mathrm{A}^{\circ} \mathrm{BBB}^{\circ} \mathrm{B}^{\circ}\right)$ y de $T$. caerulea $(\mathrm{CC})$. Los resultados obtenidos en el presente trabajo sugieren que $T$. joelii posee un genoma diferente de los de $T$. aurelii y $T$. caerulea, por lo que se propone para esa especie la fórmula genómica DD.

Los bivalentes observados en los dos híbridos se deberían a apareamiento autosindético entre los cromosomas de $T$. aurelii, por ser esta especie un alooctoploide segmentario. El apareamiento se produciría entre los cromosomas de los genomas homeólogos de A o de B. Como en los dos híbridos la configuración más frecuente fue de 17 I + 4 II, es probable que en ambos híbridos estén involucrados los mismos cromosomas en la formación de los bivalentes. Es interesante destacar que en el progenitor $T$. aurelii los cromosomas homeólogos no se aparean, pero sí lo hacen en los híbridos, como ya fue observado anteriormente (Fernández y Arbo, 1993a). Los trivalentes y los cuadrivalentes probablemente se formen por translocaciones entre los genomas A y B.

\section{Agradecimientos}

Este trabajo se llevó a cabo con el apoyo económico de la SECYT-UNNE y del CONICET. 


\section{Bibliografía}

Arbo. M. M. y A. Fernández. 1987. Cruzamientos intra e interespecíficos enTurnera, serieCanaligerae. Bonplandia 6 (1): 23-38.

Asker, S. 1980. Gametophytic apomixis. Elements and genetic regulation. Hereditas 93: 277-293.

Fernández, A. 1973. El ácido láctico como fijador cromosómico. Bol. Soc. Argen. Bot. 15 (2-3): 287-290.

Fernández. A. 1987. Estudios cromosómicos en Turnera y Piriqueta (Turneraceae). Bonplandia 6(1): 1-21.

Fernández, A. y M. M. Arbo. 1989. Relaciones genómicas entre cuatro especies diploides de Turnera con flores amarillas (Serie Canuligerae). Bonplandia 6(2): 93-109.

Fernández, A. y M. M. Arbo. 1990. Gametas no reducidas y relaciones genómicas en tres especies de Turnera (Turneraceae). Darwiniana 30 (1-4): 21-26.

Fernández, A. y M. M. Arbo. 1993a. Relaciones genómicas entre seis especies de Turnera (Serie Canaligerae) del
Paraguay. Candollea 48: 305-318.

Fernández, A. y M. M. Arbo. 1993b. Citogenética de híbridos entre Turnera grandidentata $(4 \mathrm{x})$ y $T$. subulata y T. scabra (2x) (Tumeraceae). Bonplandia 7 (1-4): 119127.

Fernández, A. y M. M. Arbo. 1996. Relaciones genómicas entre las especies diploides de flores blanco-azuladas de Turnera (Serie Canaligerae). Bonplandia 9(1-2): 95102.

Harlan, J. R. y J. M. J. de Wet. 1975. On Ö. Winge and a prayer: the origins of polyploidy. Bot. Rev. 41 (4): 361390.

Solís Neffa, V. 1996. Cariotipos de especies de Turnera (Turneraceae). Bonplandia 9 (1-2): 121-127.

Solís Neffa, V.y A. Fernández. 1993. Estudios cromosómicos en especies de Turnera (Tumeraceae). Bonplandia 7 (14): 101-118.

Urban, I. 1883. Monographie der familie der Turneraceen. Jahrb. Königl. Bot. Gart. Berlin 2: 1-152. 